\title{
The intravenous glucose tolerance and postprandial glucose tests may present different responses in the evaluation of obese dogs
}

\author{
Márcio Antonio Brunetto ${ }^{1}$, Fabiano César Sá ${ }^{1}$, Sandra Prudente Nogueira ${ }^{1}$, Márcia de Oliveira Sampaio \\ Gomes $^{1}$, Amanda Gullo Pinarel ${ }^{1}$, Juliana Toloi Jeremias ${ }^{1}$, Francisco José Albuquerque de Paula ${ }^{2}$ and \\ Aulus Cavalieri Carciofi ${ }^{1 *}$ \\ ${ }^{1}$ Veterinary Medicine and Surgery Department, College of Agrarian and Veterinarian Sciences (FCAV), São Paulo State \\ University - UNESP, Via de Acesso Professor Paulo Donato Castellane, s/n, Jaboticabal, SP 14884-900, Brazil \\ ${ }^{2}$ Division of Endocrinology and Metabolism, Department of Internal Medicine, University of São Paulo -USP, Av. \\ Bandeirantes 3900, Ribeirão Preto, SP 14049-900, Brazil
}

(Received 20 October 2010 - Revised 15 December 2010 - Accepted 28 January 2011)

\begin{abstract}
The present study compared the intravenous glucose tolerance test (IVGTT) and the glucose postprandial response (GPPR) test for the evaluation of glucose metabolism in obese dogs. A total of ten owned obese dogs (body condition score (BCS) of 9; fat mass, 45.7 (sem 1.51)\%) were used. These dogs had their weight reduced by $20 \%$ (BCS, 8; fat mass, 33.5 (sem 1.92) \%; P<0.001), designated as weight-reduced (WR) group. A control group of ten Beagle dogs was also included (BCS, 4.5; fat mass, 18.3 (sEM 1.38) \%; $P<0 \cdot 01$ ). Glucose tolerance was measured by two methods: IVGTT (infusion of $0.5 \mathrm{~g}$ of glucose/kg body weight) and GPPR (consumption of cooked rice to achieve $6 \mathrm{~g}$ of $\mathrm{starch} / \mathrm{kg}$ body weight). When using the IVGTT, the area under the curve (AUC) for glucose and maximum glycaemia were higher for obese dogs compared with controls $(P<0.05)$, with intermediate results for the WR group (P>0.05). Basal insulin, insulin response peak, insulinogenic index and the AUC for insulin increment from 0 to $15 \mathrm{~min}$ and from 60 to 120 min were higher for the obese group $(P<0.05)$, while the WR group and control dogs showed similar results $(P>0.05)$. When using the GPPR test, the AUC for insulin increment from 0 to 120 min was higher for the obese group compared with the control group $(P<0.05)$ and intermediate for the WR group $(P>0.05)$. However, the AUC for insulin increment from 120 to 360 min was similar between the obese and WR groups $(P>0.05)$, while it was lower for the control group $(P<0.05)$. The IVGTT showed that the loss of $20 \%$ body weight resulted in an improvement of glucose control with reduced insulin secretion, and these same WR dogs showed higher insulin secretion with values similar to those of obese dogs when the GPPR test was used.
\end{abstract}

Key words: Glycaemia: Insulin: Obesity

Recent studies have suggested mechanisms for the relationship between excessive body fat and many degenerative diseases. It has been found that adipose tissue, once considered to be physiologically inert, is an active producer of hormones, such as leptin and resistin, and numerous cytokines ${ }^{(1)}$. The persistent low-grade inflammation secondary to obesity is thought to play a causal role in chronic problems, including orthopaedic disease, insulin resistance, diabetes mellitus in cats, abnormalities in circulating lipid profiles, cardiorespiratory disease, urinary disorders, reproductive disorders and others ${ }^{(2)}$.

An important aspect of the negative effects of obesity may be due to glucose intolerance and insulin resistance ${ }^{(3)}$. Both conditions can be experimentally induced in dogs by dietary manipulation causing obesity. Furthermore, lifelong dietary energy restriction has been shown to improve insulin sensitivity and glucose tolerance of dogs and has been associated with reduced disease conditions and greater longevity ${ }^{(3)}$. However, few studies have examined this condition in naturally owned obese dogs, as most studies were conducted under laboratory conditions. Further studies are required to clarify the relationship between insulin resistance in naturally occurring canine obesity and the occurrence of degenerative diseases. Insulin resistance is characterised by elevated plasma insulin and exacerbated insulin responses to intravenous glucose infusion. The gold standard method to study this condition has been the hyperinsulinaemic, isoglycaemic

Abbreviations: AUC, area under the curve; BCS, body condition score; GPPR, glucose and insulin postprandial response; IVGTT, intravenous glucose tolerance test; WR, weight-reduced.

Presented as poster number 067 .

*Corresponding author: A. C. Carciofi, fax +55 163203 1226, email aulus.carciofi@gmail.com 
glucose clamp ${ }^{(4)}$. However, this method requires general anaesthesia, and its use is inconvenient for owned dogs.

Thus, the present study compared two methods - the glucose and insulin postprandial response (GPPR) test and the intravenous glucose tolerance test (IVGTT) - for measuring glucose tolerance and insulin sensitivity in owned obese dogs before and after weight loss.

\section{Materials and methods}

The assay was conducted at the Laboratory of Research on Nutrition and Nutritional Diseases of Dogs and Cats, UNESP, Jaboticabal, Brazil. All procedures were approved by the Ethics and Animal Welfare Committee (Protocol no. 017665-07). All clinical procedures performed were for the direct benefit of the dogs used in the study, and the owners of all participating animals gave written informed consent.

\section{Animals}

The study included three groups. The first group was composed of ten owned obese dogs (three Labrador retrievers, one Beagle, one Rotweiller, one Dachshund and four mixed breeds; two dogs were male and eight were female; all dogs were neutered) with a median age of 83 months (minimum-maximum 19-166), a body condition score (BCS) of 9, a mean fat mass of 45.7 (SEM 1.51) \% and a mean lean mass of 54.3 (SEM 1.51)\%. All dogs were referred to the Clinical Nutrition Service, UNESP, Jaboticabal, Brazil, for the investigation and management of obesity or obesity-related disorders. These dogs, according to body-weight records, had been in a static phase of obesity for more than 12 months. Dogs were enrolled if they were systemically well at the physical examination, and no significant abnormalities were detected on their complete blood count, serum biochemical analysis, thyroid hormone analysis and urinalysis. The second group was composed of these same dogs after a controlled loss of $20 \%$ of their initial body weight; this group was designated as weight-reduced (WR) group (BCS, 8; fat mass, 33.5 (SEM 1.92) \% and lean mass, 66.5 (SEM $1.92) \%(P<0.001)$; no changes in lean mass (in $\mathrm{kg}$ ) $(P>0.05))$. A control group of ten Beagle dogs was also included $(5.0$ (SEM 0.75 ) years old; BCS, 4.5; fat mass, 18.3 (sem 1.38$) \%$ and lean mass, $81.7 \%(P<0 \cdot 01)$ ).

\section{Weight-loss protocol}

A BCS was assigned to each dog using a 9-integer system, as described previously ${ }^{(5)}$, and body composition was determined by the ${ }^{2} \mathrm{H}$ isotope dilution technique ${ }^{(6)}$. The dogs were fed restricted amounts, which corresponded to $60 \%$ of their maintenance energy requirement ${ }^{(7)}$ that was calculated for their target weight. The target weight was standardised as the current weight minus $20 \%$. The diet used in the study was presented on a DM basis: $32 \%$ of crude protein, $9 \%$ of fat, $22 \%$ of dietary fibre and $11.7 \mathrm{MJ} / \mathrm{g}$ of metabolisable energy determined in vivo (Guabi Natural Obesos; Mogiana Alimentos, Campinas, Brazil). Each owner received a measuring cup adjusted to his animal to make it easier to give the correct daily amount of food, which was divided into two or three meals according to their convenience. Other recommendations included daily walks and no treats or other foods during the treatment.

\section{Glucose tolerance and postprandial response tests}

The IVGTT was performed after $12 \mathrm{~h}$ of fasting. Each dog was aseptically catheterised using a peripheral intravenous catheter (Angiocath $20 \mathrm{GA} \times 1.16$ in.; Becton-Dickinson, Franklin Lakes, NJ, USA) placed into the cephalic vein. Blood samples were taken before (baseline sample, time 0 ) and 1, 2.5, 5.0, 7.5, 10, $15,30,45,60,90$ and $120 \mathrm{~min}$ after the infusion of $0.5 \mathrm{~g}$ of glucose/kg body weight. For the GPPR test, the same procedure for catheter placement was used after fasting for $12 \mathrm{~h}$. Blood samples were taken pre-feeding (baseline sample, time 0 ) and 5, 10, 15, 30, 60, 120, 180, 240, 300 and $360 \mathrm{~min}$ after the consumption of a defined amount of cooked rice that was enough to achieve the ingestion of $6 \mathrm{~g}$ of starch $/ \mathrm{kg}$ body weight. Blood was always collected at the same time, beginning at 10.00 hours. Each sample $(2 \mathrm{ml})$ was collected in a Na-heparin tube and centrifuged ( $378 \boldsymbol{g}$ for $5 \mathrm{~min}$ ), and plasma was separated into two Eppendorf tubes. Plasma samples for glucose measurement were kept under refrigeration $\left(4^{\circ} \mathrm{C}\right)$ for a maximum of $2 \mathrm{~h}$ before analysis; plasma samples for insulin measurements were frozen $\left(-20^{\circ} \mathrm{C}\right)$ for a maximum of 6 months before they were analysed.

\section{Laboratorial analyses}

Plasma glucose concentrations were determined by the glucose oxidase test (glucose-specific enzyme method; Labtest Diagnóstica S.A., Lagoa Santa, Brazil) using a semi-automatic glucose analyser (Labquest model BIO-2000; Labtest Diagnóstica S.A.). Plasma insulin levels were measured by RIA using a commercially available kit (Siemens, Los Angeles, CA, USA) that has already been validated for dogs. The intra-assay $\mathrm{CV}$ for insulin was $3.45 \%$, and the standard error was $0.83 \mathrm{pmol} / \mathrm{l}$.

\section{Calculations and statistical analyses}

Calculation procedures used for IVGTT data interpretation have been described in the literature ${ }^{(8)}$. Data obtained during the GPPR test were analysed as described by Carciofi et $a l .{ }^{(9)}$. The integrated area under the curve (AUC) for glucose and insulin absolute values and increments were calculated by the trapezoidal method using ORIGIN software (version 6.0; Microcal Software, Inc., Northampton, MA, USA). Glucose and insulin curves were analysed by repeated-measures ANOVA. Glucose and insulin parameters and the AUC were analysed by paired (obese $\times \mathrm{WR}$ ) or non-paired $t$ tests. Variables not complying with ANOVA assumptions were analysed by the Wilcoxon test. Data were analysed using the general linear model procedure of the Statistical Analysis Systems statistical software package version 9.0 (SAS Institute, Cary, NC, USA). Results were considered to be significantly different when $P<0 \cdot 05$. 
Table 1. Results of the intravenous glucose tolerance test in obese dogs, in these same dogs after a loss of $20 \%$ of body weight (weight-reduced (WR) dogs) and in a group of control non-obese dogs

(Medians and ranges)

\begin{tabular}{|c|c|c|c|c|c|c|}
\hline & \multicolumn{6}{|c|}{ Dogs } \\
\hline & \multicolumn{2}{|c|}{ Obese $(n 10)$} & \multicolumn{2}{|r|}{ WR $(n 10)$} & \multicolumn{2}{|c|}{ Control $(n 10)$} \\
\hline & Median & Minimum-maximum & Median & Minimum-maximum & Median & Minimum-maximum \\
\hline$K(\%)$ & $3 \cdot 6^{a}$ & $2 \cdot 49-6 \cdot 69$ & $2 \cdot 6^{\mathrm{a}}$ & $0.30-14 \cdot 62$ & $3 \cdot 1^{\mathrm{a}}$ & $2 \cdot 35-6 \cdot 62$ \\
\hline$T_{1 / 2}(\min )$ & $19 \cdot 5^{\mathrm{a}}$ & $10 \cdot 35-27 \cdot 79$ & $26 \cdot 7^{\mathrm{a}}$ & $4.73-29.87$ & $19 \cdot 4^{a}$ & $10 \cdot 46-29 \cdot 41$ \\
\hline$\Delta / / \Delta G$ & $0.14^{\mathrm{a}}$ & $0.09-0.17$ & $0 \cdot 10^{\mathrm{b}}$ & $0.09-0.12$ & $0.09^{b}$ & $0.03-0.56$ \\
\hline $\operatorname{IRP}(\mu \mathrm{Ul} / \mathrm{ml})$ & $71.9^{a}$ & $56 \cdot 40-108 \cdot 70$ & $18.0^{\mathrm{b}}$ & $15.00-73.00$ & $31.9^{\mathrm{b}}$ & $13.40-15.00$ \\
\hline IRT (min) & $15 \cdot 0^{\mathrm{a}}$ & $1.00-30.00$ & $10 \cdot 0^{\mathrm{ab}}$ & $7.50-15.00$ & $1 \cdot 7^{\mathrm{b}}$ & $0.00-15.00$ \\
\hline
\end{tabular}

$K$, percentage of glucose disappearance; $T_{1 / 2}$, time for glucose concentration to reduce to half; $\Delta / / \Delta G$, insulinogenic index; IRP, insulin response peak; IRT, insulin response peak (in time).

a,b Values within a row with unlike superscript letters were significantly different by Wilcoxon's test $(P<0.05)$.

\section{Results}

After their regimens, dogs lost $22 \cdot 0$ (SEM 1.1)\% of their initial body weight in a mean rate of 0.70 (SEM 0.05 ) \% of their body weight per week. The mean reduction in body fat mass was 27 (SEM 2.0)\%, with no reduction in lean mass (in kg) $(P>0 \cdot 05)$.

In the IVGTT, higher glycaemia was found for obese dogs than for control dogs at times 1.0, 2.5 and 5.0 min $(P<0.05)$. The AUC for glucose, maximum and mean glycaemia were higher for obese dogs than for control dogs $(P<0.05)$ and intermediate for WR dogs $(P>0 \cdot 05)$. Basal insulin concentration was higher for obese dogs (29.59 (sEm 7.64) pmol/1) than for WR $(13.4$ (SEM 7.8) pmol/l) and control (10.2 (SEM $5.97) \mathrm{pmol} / \mathrm{l})$ dogs $(P<0 \cdot 05)$. Insulin response peak, insulinogenic index, the AUC for insulin increment between 0 and $15 \mathrm{~min}$ and between 60 and $120 \mathrm{~min}$ were higher for obese dogs $(P<0.05)$ than for WR or control dogs that were not different from each other $(P>0.05)$. Data are presented in Table 1.

In the GPPR test, obese dogs (903 (SEM 61.0) $\mathrm{mg} / \mathrm{l}$ ) presented higher basal glycaemia than WR (67 (sEm 33.3) $\mathrm{mg} / \mathrm{l})$ and control $(683($ SEM 33.7$) \mathrm{mg} / \mathrm{l})$ dogs $(P<0.05)$. Results are illustrated in Fig. 1. The AUC for glucose from 0 to 360 , from 0 to 120 and from 120 to 360 was higher for obese dogs $(P<0.05)$ than for WR and control dogs. The AUC for insulin increment between 0 and $120 \mathrm{~min}$ was higher for obese dogs than for control dogs $(P<0.05)$ and intermediate for WR dogs $(P>0.05)$, although the delayed secretion (AUC for insulin increment between 120 and $360 \mathrm{~min}$ ) was similar for obese and WR dogs $(P>0.05)$, with higher values than for control dogs $(P<0 \cdot 05)$.

\section{Discussion}

Obese dogs have insulin resistance, as determined during the IVGTT by elevated fasting plasma insulin concentration, insulinogenic index, insulin response peak and delayed insulin secretion (AUC for insulin increment from 60 to $120 \mathrm{~min}$ ). The loss of $27 \%$ of fat mass (WR dogs) resulted in significant improvement in carbohydrate metabolism; in the IVGTT, WR dogs showed results similar to those of control dogs.
Alterations in carbohydrate metabolism were also evident in obese dogs in the GPPR test, with high glucose and insulin postprandial levels. However, the induced weight loss was not enough to reverse these alterations; in the GPPR test, WR dogs still showed elevated delayed insulin secretion with values similar to those of obese dogs.
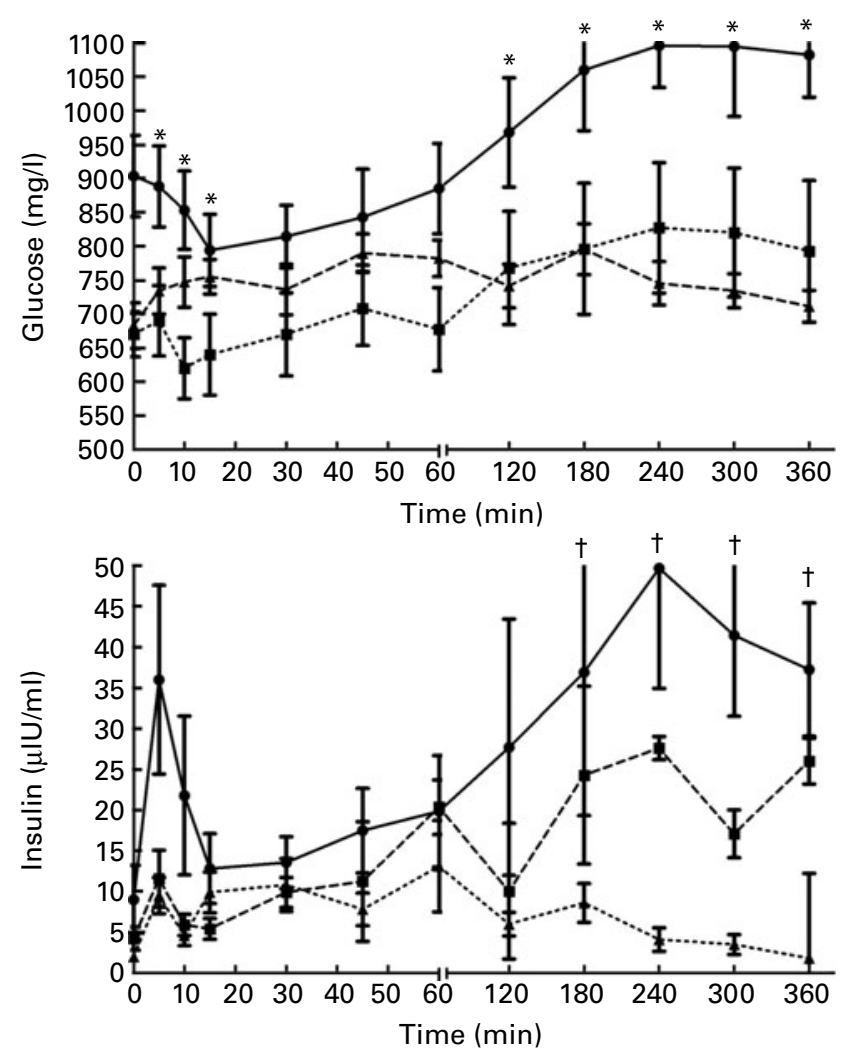

Fig. 1. Results of the glucose and insulin postprandial response of obese dogs (- - $n$ 10), in these same dogs after a loss of $20 \%$ of body weight (weight-reduced (WR, - - -) dogs, $n 10$ ) and in a group of control non-obese dogs $(-\boldsymbol{\Delta}-, n 10)$. Values are means, with their standard errors represented by vertical bars. * Mean values were significantly different with higher glycaemic values for obese dogs than for WR and control dogs $(P<0.05)$. † Mean values were significantly different with higher insulinaemia for obese dogs than for control dogs $(P<0.05)$, presenting intermediary levels for WR dogs $(P>0.05)$. 
Other authors $^{(3,8,10)}$ have already shown glucose and insulin disturbances in obese dogs using the IVGTT, verifying a positive relationship among adiposity and insulin secretion $(P<0.05)$. However, we did not find comparable studies that evaluated the effect of the weight loss of owned obese dogs on insulin resistance or glucose tolerance. Another problem in comparing the present results with those of others is the amount of body fat of dogs studied. We studied grossly obese animals ( $45.7 \%$ of fat mass), whereas obesity induced in a laboratory situation is much milder ${ }^{(11,12)}$. Even after $22 \%$ of body-weight loss, WR dogs presented a mean BCS of 8 and $33.5 \%$ of fat mass, so the animals were far from the ideal body condition. The amount of body fat mass and possibly the time that the animals remained obese are points that need to be adequately considered in studies of obesity in dogs.

Interpreting the GPPR test, we observe that the results between 120 and $360 \mathrm{~min}$ are very important to differentiate groups. Obese animals presented a remarkable elevation in glucose and insulin serum concentrations in this interval. No other studies were found to compare with the present findings. Considering the complex endocrine control of food digestion and whole-body metabolism performed by the intestine producing and releasing dozens of peptides and hormones $^{(7)}$, using the postprandial response to evaluate carbohydrate metabolism, appears to be much more reasonable.

\section{Conclusion}

The IVGTT and GPPR test present different results, and maybe different responses. While the IVGTT showed that the loss of $20 \%$ body weight resulted in the improvement of glucose control with reduced insulin secretion, these same dogs at the GPPR test showed higher insulin secretion with values similar to those of obese dogs.

\section{Acknowledgements}

M. A. B., M. d. O. S. G. and A. C. C. conceived the present study and drafted the manuscript. M. A. B was the main executor, and F. C. S., S. P. N., A. G. P. and J. T. J. contributed to the execution of the present study. F. J. A. P. was responsible for insulin analysis. All authors contributed to the critical revision of the manuscript. The study was supported by FAPESP (process 07/08298-8), CNPq and Mogiana Alimentos (Guabi). None of the authors has any conflicts of interest.

\section{References}

1. Gayet C, Bailhache E, Dumon H, et al. (2004) Insulin resistance and changes in plasma concentration of TNF alfa, IGF 1 , and NEFA in dogs during weight gain and obesity. J Anim Physiol Anim Nutr 88, 157-165.

2. Sowers M, Jannausch M, Stein E, et al. (2002) C reative protein as a biomarker of emergent osteoarthritis. Osteoarthritis Cartilage 10, 595-601.

3. Larson BT, Lawler DF, Spitznagel EL, et al. (2003) Improved glucose tolerance with lifetime diet restriction favorably affects disease and survival in dogs. J Nutr 133, 2887-2892.

4. German AJ, Hervera M, Hunter L, et al. (2009) Improvement in insulin resistance and reduction in plasma inflammatory adipokines after weight loss in obese dogs. Domest Anim Endocrinol 27, 214-226.

5. Laflamme D (1997) Development and validation of a body condition score system for dogs. Canine Practice 22, 10-15.

6. Ferrier L, Robert P, Dumon H, et al. (2002) Evaluation of body composition in dogs by isotopic dilution using a lowcost technique, Fourier-transform infrared spectroscopy. $J$ Nutr 132, 1725S-1727S

7. National Research Council (US) Ad hoc Committee on Dog and Cat Nutrition (2006) Nutrient Requirements of Dogs and Cats. Washington, DC: The National Academies Press.

8. Mattheeuws D, Rottiers R, Kaneko JJ, et al. (1984) Diabetes mellitus in dogs: relationship of obesity to glucose tolerance and insulin response. Am J Vet Res 45, 98-103.

9. Carciofi AC, Takakura FS, de-Oliveira LD, et al. (2008) Effects of six carbohydrates sources on dog diet digestibility and post-prandial glucose and insulin response. J Anim Physiol Anim Nutr 92, 326-336.

10. Mattheeuws D, Rottiers R, Baeyens D, et al. (1984) Glucose tolerance and insulin response in obese dogs. $\mathrm{J} \mathrm{Am} \mathrm{Vet}$ Med Assoc 20, 287-293.

11. Gayet C, Leray V, Saito M, et al. (2007) The effects of obesityassociated insulin resistance on mRNA expression of peroxisome proliferator-activated receptor $\gamma$ target genes in dogs. Br J Nutr 98, 497-503.

12. Respondek F, Swanson KS, Belsito KR, et al. (2008) Shortchain fructooligosaccharides influence insulin sensitivity and gene expression of fat tissue in obese dogs. $J$ Nutr 138, 1712-1718. 